Emmer, Martin; Wolling, Jens:

Online-Kommunikation und politische Öffentlichkeit

Original erschienen in:

Schweiger, Wolfgang; Beck, Klaus (Hrsg.): Handbuch Online-Kommunikation. Wiesbaden : VS, Verl. für Sozialwiss. - 2010, S. 36-58.

ISBN (print): 978-3-531-17013-8

ISBN (online): 978-3-531-92437-3

DOI:

10.1007/978-3-531-92437-3_2

URL: http://dx.doi.org/10.1007/978-3-531-92437-3_2

[Gesehen: 18.12.2015] 


\title{
Online-Kommunikation und politische Öffentlichkeit
}

\begin{abstract}
Veränderungen politischer Öffentlichkeit gehören zu den in der Literatur intensiv diskutierten Folgen des Internets und der Online-Kommunikation. Ein Grund hierfür sind die besonderen Strukturmerkmale von Online-Kommunikation, die die Realisierung direktdemokratischer Bürgerbeteiligung, die unter den Rahmenbedingungen klassischer Massenmedien in repräsentativen Demokratien nur unvollkommen möglich ist, plötzlich greifbar erscheinen lassen. Während die theoretische Debatte bisher die grundsätzlichen Chancen und Risiken der Online-Kommunikation für den öffentlichen Diskurs und die politische Kommunikation herausgearbeitet hat, liegen bislang nur begrenzt verlässliche empirische Ergebnisse zu den möglichen Folgen des Netzes in diesem Bereich vor. Insgesamt lassen sich bestimmte Verschiebungen in der politischen Mediennutzung, in der Themenwahrnehmung und im öffentlichen Diskurs beobachten. Diese Veränderungen folgen dabei eher einem evolutionären als einem revolutionären Schema. Es überwiegen Befunde, die aus normativer Perspektive, positive' Folgen konstatieren, wobei hinsichtlich der langfristigen Entwicklung politischer Öffentlichkeit auf Basis der bisherigen Forschung noch kaum Aussagen getroffen werden können.
\end{abstract}

\section{Schlagwörter}

Öffentlichkeit, politische Kommunikation, Medienwirkung, öffentliche Meinung, AgendaSetting, Priming, Framing, Deliberation, politische Mobilisierung, Politikverdrossenheit, Mediamalaise, Partizipation

\section{Problemaufriss: Internet und politische Öffentlichkeit}

Von Beginn der Internet-Ära an stand bei der Diskussion über die Chancen der OnlineKommunikation deren vermutete politische, gesellschaftsverändernde Kraft im Zentrum. So formulierte Al Gore im März 1994 seine Vision von der Bedeutung der von ihm politisch geförderten, auf dem Internet basierenden ,Global Information Infrastructure“ (GII): 
"The GII will not only be a metaphor for a functioning democracy, it will in fact promote the functioning of democracy by greatly enhancing the participation of citizens in decision-making. And it will greatly promote the ability of nations to cooperate with each other. I see a new Athenian Age of democracy forged in the fora the GII will create."

Aus dieser wie auch aus zahlreichen ähnlichen Aussagen wird zum einen die Bedeutung des Internets und seiner Kommunikationsoptionen (,Foren der Demokratie') für die politische Öffentlichkeit und den politischen Prozess deutlich, zum anderen zeigt sich daran die starke normative Komponente der Debatte um das demokratische Potenzial von Online-Kommunikation, die sich primär an einem Modell deliberativer Öffentlichkeit orientiert. Im Modell der deliberativen Öffentlichkeit, das sich theoretisch auf Habermas (1991) zurückführen lässt, ist die herrschaftsfreie und intensive Teilhabe möglichst vieler Bürger, beruhend auf einer weitgehenden Gleichheit der Teilnahmechancen am politischen Diskurs, ein zentrales Qualitätskriterium demokratischer Öffentlichkeit (Peters 2002: 24-25). Gerhards und Neidhardt (Gerhards 1998: 269; Gerhards \& Neidhardt 1993: 60-61) definieren Öffentlichkeit auf dieser Basis als ein Kommunikationssystem, das es Akteuren erlaubt, Informationen und Meinungen auszutauschen, und das durch seine Eigenschaften die Herausbildung öffentlicher Meinungen zu spezifischen Problemen ermöglicht. In diesem Öffentlichkeitsverständnis liegt die Betonung auf der Validierung der politischen Entscheidung. Darüber hinaus werden der Öffentlichkeit noch weitere demokratierelevante Funktionen zugesprochen (Imhof 2003), wie die Herstellung von Transparenz (z.B. durch Thematisierung, Agenda-Setting) oder die möglichst enge Bindung der Entscheidungsträger (Orientierungsfunktion) an die öffentliche Meinung (Responsivität).

Theoretisch lässt sich das Konstrukt der Öffentlichkeit nicht von den Prinzipien demokratischer Gesellschaftsordnungen trennen: Bereits in den Anfängen der Demokratie in den griechisch-antiken Stadtstaaten war die Möglichkeit der öffentlichen, allen Bürgern zugänglichen Diskussion gemeinschaftsrelevanter Fragen ein konstituierendes Element von Demokratie (Guggenberger 1995). Im Zuge der Aufklärung wurde dann die Freiheit, die eigene Meinung in öffentlicher Rede ungehindert zu äußern, nicht nur zu einem Zeichen der Mündigkeit der Bürger, sondern auch als Voraussetzung anerkannt, auf deren Basis vernünftige politische Entscheidungen getroffen werden können (Imhof 2003). Die Entscheidung des Bundesverfassungsgerichts, die Freiheit der Medien als Demokratie konstituierend zu deklarieren, bewegt sich in dieser gedanklichen Tradition. Sie beruht auf der Erkenntnis, dass Öffentlichkeit längst nicht mehr nur in der individuellen Interaktion auf der Mikroebene und in der Versammlungsöffentlichkeit, sondern in modernen Massendemokratien vor allem auch massenmedial entsteht.

Diese zunehmende Bedeutung der Massenmedien für die Herstellung von Öffentlichkeit stellt für die Legitimität der Demokratie jedoch eine erhebliche Herausforderung dar. Durch die Gatekeeper-Funktion der Massenmedien ist der Zugang zur Öffentlichkeit an die Logik der massenmedialen Selektion geknüpft. Für Akteure, Themen, Positionen, die nicht in diese Logik passen, ist es schwierig, öffentliche Aufmerksamkeit zu erlangen. Damit ist die Transparenzfunktion von Öffentlichkeit gefährdet. Auch die Orientierungsfunktion der Massenmedien ist 
aus verschiedenen Gründen zu problematisieren: Zum einen ist hier der Einfluss ökonomischer Interessen zu nennen, der dazu führt, dass nicht allein die Relevanz darüber entscheidet, ob eine Meinung medial verbreitet wird. Zum anderen ist aber vor allem die These, dass Massenmedien aus strukturellen Gründen überhaupt nicht in der Lage sein können, eine auf rationalem Diskurs beruhende öffentliche Meinung herzustellen, ein schwerwiegender Einwand. Demzufolge kann aufgrund der fehlenden Möglichkeiten zur interaktiven, deliberativen Debatte in den Massenmedien eine rationale Validierung der Argumente nicht stattfinden und damit auch keine öffentliche Meinung entstehen.

Vor diesem Hintergrund sind die hohen Erwartungen nachvollziehbar, dass die OnlineKommunikation mit ihren niedrigen Zugangshürden und ihrem hohen Interaktionspotenzial nicht nur zur politischen Transparenz beiträgt, sondern vor allem auch die deliberative Validierung politischer Entscheidungen vorantreibt und damit die Basis für eine engere Bindung der Entscheidungsträger an die öffentliche Meinung geschaffen wird. Allerdings wurden solche Erwartungen schon häufig an Medieninnovationen herangetragen und bisher selten erfüllt (ein Beispiel sind die partizipatorischen Erwartungen Brechts an das Radio: Brecht 1975).

\section{Abbildung 1: Internet und politische Kommunikation - Forschungsfelder, Annahmen und forschungsleitende Fragen}

\begin{tabular}{l}
\hline \multicolumn{4}{|c|}{ Zugang zum Internet } \\
\hline \begin{tabular}{|l|c|c|c|}
\hline $\begin{array}{l}\text { Potenziale } \\
\text { des Ange- } \\
\text { bots }\end{array}$ & Egalität im Zugang & $\begin{array}{c}\text { Diskursivität der } \\
\text { Auseinandersetzung }\end{array}$ & $\begin{array}{c}\text { Unabgeschlossenheit } \\
\text { des Zugangs }\end{array}$ \\
\cline { 2 - 4 } & \multicolumn{3}{|c|}{ Vielfalt der Optionen? } \\
\hline \begin{tabular}{|l|c|c|c|} 
Formen \\
der Nut- \\
zung
\end{tabular} & Politische Information & $\begin{array}{c}\text { Politische Interpersonale } \\
\text { Interaktion }\end{array}$ & Politische Partizipation \\
\cline { 2 - 4 } & \multicolumn{3}{|c|}{ Intensivierung der Nutzung? } \\
\hline \multirow{2}{*}{$\begin{array}{l}\text { Folgen der } \\
\text { Nutzung }\end{array}$} & \begin{tabular}{c} 
Wissen \\
Agenda Setting \\
\cline { 2 - 4 }
\end{tabular} & $\begin{array}{c}\text { Fragmentierung } \\
\text { Orfentliche Meinung }\end{array}$ & $\begin{array}{c}\text { Priming } \\
\text { Persuasion }\end{array}$ \\
\cline { 2 - 4 } & \multicolumn{2}{|c|}{ Auswirkungen auf die Politik (polity, politics, policy)? } \\
\hline
\end{tabular}
\end{tabular}

In diesem Beitrag sollen die aus Bürgerperspektive relevanten Aspekte politischer Öffentlichkeit unter den Bedingungen der Online-Kommunikation umrissen werden (Abbildung 1): Wie verändern sich grundsätzlich die politischen Kommunikationsoptionen für die Bürger? Welche ,Medien' stehen ihnen in ihrem Nutzungsalltag zur Verfügung und wie gehen sie mit den neuen Kommunikationsoptionen um? Welche Rolle spielen heute Online- und Offline-Medien für die öffentliche Kommunikation der Bürger? Welche Effekte hat das veränderte Medienumfeld auf 
den öffentlichen Diskurs? Welche politischen Medienwirkungen kann man der OnlineKommunikation zuschreiben?

Zur Beantwortung dieser Fragen soll in einem ersten Abschnitt theoretisch erörtert werden, wie sich aus Bürgerperspektive die Rahmenbedingungen der Medienöffentlichkeit durch die Optionen der Online-Kommunikation konkret verändern. Anschließend wird der aktuelle Forschungsstand zur Nutzung des Netzes durch Bürger für ihre Beteiligung am politischen Prozess präsentiert. In einem dritten Abschnitt werden die bisherigen Erkenntnisse zu den weiter reichenden Folgen und Wirkungen der Online-Kommunikation auf die Bürger und den öffentlichen Diskurs vorgestellt.

\section{Theoretische Grundlagen: Strukturelle Veränderungen von Öffentlichkeit durch das Internet}

Es ist theoretisch gut begründbar, dass Online-Kommunikation die Kommunikationsmöglichkeiten der Bürger deutlich erweitert. Deswegen ist es auch plausibel, dass dies Folgen für die Form und den Inhalt ihrer öffentlichen politischen Kommunikation haben könnte. Neben Autoren, die die Chancen der Online-Kommunikation für die Demokratie betonen (Browning 1995; Scammell 2000), finden sich andere, die mögliche Probleme und negative Konsequenzen des Netzes für die politische Kommunikation identifizieren (Noam 1999; Tsaliki 2002). Die immer wieder thematisierten praktischen Probleme mit Inhalten (z.B. politischer Extremismus) und den Strukturen des Netzes (Spam, Datenschutz etc.) belegen, dass Netzkommunikation Risiken mit sich bringt, die auch demokratische Gesellschaften vor die Herausforderung stellen, kontrollierend und regulierend einzugreifen.

Die Möglichkeit für Bürger, sich aktiv am öffentlichen Diskurs über gesellschaftlich relevante Probleme zu beteiligen, ist nach dem oben dargelegten Verständnis von Öffentlichkeit eine Voraussetzung für ihre Wirksamkeit im politischen Prozess. Da allein aufgrund der quantitativen Asymmetrie zwischen politischem Personal und Bürgern eine persönliche Kommunikation in „Encounter-“ bzw. „Begegnungsöffentlichkeiten“ sowie eine Ebene darüber in Versammlungsöffentlichkeiten nur in Ausnahmefällen zu realisieren ist, spielte bisher die „Medienöffentlichkeit" (zur Unterscheidung der Ebenen siehe Neidhardt 1994) die wichtigste Rolle. Hier findet sich ein erster Ansatzpunkt für mögliche strukturelle Veränderungen von Öffentlichkeit durch Online-Kommunikation: Durch die computerbasierte Kommunikations-Infrastruktur wird eine Vielfalt an Medien ermöglicht, deren Hybridcharakter (Höflich 1997) die scharf gezogenen Grenzen traditioneller Kommunikationsformen auflöst und eine Vielzahl neuer Formen der Kommunikation ermöglicht. Diese können unter Umständen auch helfen, normative Ansprüche an eine möglichst breite und intensive Beteiligung der Bürger einzulösen. Erstes zentrales Merkmal von Öffentlichkeit ist in diesem Zusammenhang die Unabgeschlossenheit des Publikums (Habermas 1962: 52-53) - grundsätzlich kann jeder Teil des Publikums sein, es gibt keine definierte Mitgliedschaft (Gerhards \& Neidhardt 1993: 61). Man kann diese Anforderung auch 
als freien Zugang zur Teilhabe am öffentlichen Diskurs verstehen, der unter den Bedingungen der klassischen massenmedialen Öffentlichkeit vielfach beschränkt war und in der Regel indirekt über professionelle Gatekeeper erfolgte. In Online-Umgebungen dagegen kann dieser Zugang nicht nur theoretisch, sondern auch praktisch deutlich erleichtert werden.

Verknüpft mit der Unabgeschlossenheit ist zweitens die Egalität der am öffentlichen Diskurs beteiligten Akteure (Gerhards \& Neidhardt 1993: 61): Debatten in der Öffentlichkeit sollen nicht an spezielle Rollenanforderungen, an Expertenwissen oder sozialen Status geknüpft sein. Einzige Voraussetzung ist die Beherrschung und Verwendung sprachlich-kommunikativer Alltagsfertigkeiten. Auch hier gibt es Autoren, die der Online-Kommunikation durch die Herrschaftsfreiheit und niedrige Zutrittsschwellen zu zahlreichen medialen Äußerungsformen einen positiven Einfluss auf öffentliche Kommunikation zuschreiben (Brants 2005).

Drittes zentrales Merkmal von Öffentlichkeit ist die Diskursivität der Kommunikation: Es wird mit nichts anderem als Sprache und Argumenten interagiert, andere Medien wie Macht oder Geld spielen keine Rolle (Gerhards 1995; Gerhards \& Neidhardt 1993; Habermas 1962). Ziel ist eine so gut wie möglich begründete öffentliche Meinung, die eine optimale politische Entscheidung durch das politische System legitimiert. Insbesondere hier lassen sich gegenüber klassischen Massenmedien Potenziale der Online-Kommunikation ausmachen, denn anders als Fernsehen oder Tageszeitung ermöglichen Online-Medien gleichberechtigte Interaktion zwischen Bürgern, und das im Prinzip ohne räumliche und zeitliche Beschränkungen (Brants 2005; Marschall 1998; Schweiger \& Weihermüller 2008).

Diese Potenziale des Internets auf Basis von Öffentlichkeitstheorien sind in der Literatur bereits früh beschrieben (Scherer 1998) und durchaus auch kontrovers diskutiert worden (Beck 2006; Dahlberg 2001). Inwieweit sie sich im Laufe der Verbreitung von Online-Medien jedoch auch realisieren und Öffentlichkeit, öffentliche Meinung und den politischen Prozess erkennbar verändert haben, soll im Folgenden auf Basis des Forschungsstandes zur Nutzung von OnlineKommunikation durch Bürger und zu den Wirkungen dieser neuen Kommunikationsmittel auf den politischen Diskurs vorgestellt werden. Hinsichtlich der Deskription der politikbezogenen Nutzung von Online-Kommunikation durch die Bürger liegt der Schwerpunkt auf Deutschland, hinsichtlich der verschiedenen Wirkungen wird jedoch der internationale Forschungsstand referiert, da sich zum einen die Forschungslage in Deutschland als unzureichend darstellt und da zum anderen die Annahme plausibel erscheint, dass die grundsätzlichen Wirkprozesse kulturübergreifend Gültigkeit besitzen. 


\section{Forschungsstand: Nutzung politischer Online-Angebote durch die Bürger}

\subsection{Grundfragen und Entwicklung der Nutzung von Online-Angeboten}

Bei allen im Folgenden vorgestellten Forschungsergebnissen und Erkenntnissen ist zu bedenken, dass die Diffusion der Online-Nutzung in unserer Gesellschaft nicht abgeschlossen ist und dass vor allem praktisch alle Kommunikationsformen im Netz einem ständigen Wandel und schneller Weiterentwicklung unterliegen. Studien aus der Frühzeit der empirischen OnlineForschung (Mitte bis Ende der 1990er-Jahre) sind aufgrund der (kleinen) Minderheit an OnlineNutzern mit ihrem speziellen Profil (jung, männlich, hoch gebildet, akademisches Umfeld) nur begrenzt verallgemeinerbar auf die heutige Gesamtheit der Internet-Nutzer und ihre OnlineNutzung. Gerade in einem solchen sich dynamisch entwickelnden Realitätsbereich sind die Ergebnisse jeder Untersuchung jeweils an den zeitlich-gesellschaftlichen Untersuchungskontext gebunden - so bezieht sich der Großteil der empirischen, politik- und wahlkampfbezogenen Online-Forschung auf die teilweise sehr spezielle US-amerikanische Politik. Deshalb muss jeweils im Einzelfall geprüft werden, inwieweit eine Verallgemeinerung oder Übertragung auf andere politische, gesellschaftliche oder historische Kontexte möglich und sinnvoll ist.

Die Nutzung des Netzes durch Bürger für Zwecke der politischen Kommunikation ist die notwendige Voraussetzung für mögliche Wirkungen von Online-Kommunikation auf die Öffentlichkeit. Strukturell unterscheiden sich Online-Angebote durch die grundsätzliche Art, wie der Kommunikationsprozess verläuft: Bei klassischen Massenmedien beschränkt sich die Nutzeraktivität auf die anfängliche Selektion einzelner Angebote. Nachfolgend werden sie dann überwiegend als Push-Angebote in Form von Komplettangeboten einzelner Medienorganisationen frei Haus (und als TV und Hörfunk größtenteils kostenlos) geliefert. Online-Angebote sind hingegen überwiegend Pull-Angebote, die sich der Nutzer in einem fortwährenden Selektionsprozess selbst aktiv sucht und zusammenstellt, auch wenn neue Formen von Push-Diensten (z.B. RSS-Feeds) im Netz an Raum gewinnen. Diese strukturelle Veränderung führt dazu, dass der Wettbewerb um die Aufmerksamkeit der Nutzer zunimmt und sich dadurch auch die politisch relevanten Online-Angebote von herkömmlichen Medienangeboten in Gestaltung und Inhalt deutlich unterscheiden (zu den grundsätzlichen Modi der Online-Kommunikation siehe den Beitrag von Beck in diesem Band).

Die Frage, welche Rolle sich Online-Medien im Gesamtangebot an Informationsquellen erobert haben, ist zumindest in quantitativer Hinsicht relativ gut erforscht. So werden seit den späten 1990er-Jahren in allen großen Rezipientenbefragungen Indikatoren erhoben, die Aufschluss darüber geben, wie intensiv die Menschen welche Kommunikationsformen in welchen Zusammenhängen nutzen. Eine besondere Rolle in Deutschland spielen hierbei, neben nur teilweise öffentlich zugänglichen Daten der kommerziellen Forschungsinstitute (z.B. Nielsen, GfK), die jährlichen Befragungen der ARD-ZDF-Arbeitsgruppe Multimedia (zuletzt van Eimeren \& Frees 
2009), welche die Entwicklung der Internet-Nutzung seit 1997 kontinuierlich mit wechselnden Schwerpunkten aufzeigen. Längerfristige Beobachtungen mit Fokus auf politischer Kommunikation und mit umfangreicherer Erhebung nicht nur von politischen Online-Nutzungs- sondern auch von individuellen Einstellungsvariablen werden für die USA seit etwa 2000 durch das Pew Internet and American Life Project des Pew Research Centers (http://people-press.org) bereitgestellt. In Deutschland bietet z.B. der ALLBUS (http://gesis.org) für manche Jahre neben politischen Einstellungs- auch Mediennutzungsindikatoren (vgl. Kaczmirek \& Raabe in diesem Band).

Die meisten Studien beschränken sich auf die Ermittlung der Nutzung des Internets für Informationszwecke. ,Nutzung' bedeutet jedoch für online-basierte Kommunikation - im Gegensatz zum klassischen Nutzungsbegriff im Rahmen der Massenkommunikationsforschung - nicht alleine (1) die mehr oder weniger passive Rezeption von Medienangeboten, sondern auch (2) aktiven Einsatz von Online-Medien zur Kontaktaufnahme und Kommunikation mit Anderen in dyadischen Beziehungen und in Kleingruppen sowie zur (3) im engeren Sinne ,öffentlichen', sprich (potenziell) öffentlich sichtbaren politischen Äußerung und zur politischen Partizipation über Online-Medien (Emmer 2005: 79-81). Eine breite Datenbasis mit explizitem Schwerpunkt auf politischer Online-Kommunikation, bei der ein solcher erweiterter Nutzungsbegriff explizit berücksichtigt wird, stellt das DFG-Projekt „Politische Online-Kommunikation“ an der TU Ilmenau seit dem Jahr 2002 zur Verfügung (http://www.politische-online-kommunikation.de).

\subsection{Online-Medien als Quelle politischer Informationen}

Die Nutzerzahlen und die Nutzungszeiten des Internets sind zwar insgesamt kontinuierlich gestiegen (van Eimeren \& Frees 2009: 335), aber das Netz wird nichtsdestotrotz von den Bürgern weniger als ein politisches Medium wahrgenommen, als dies bei anderen Medien der Fall ist: Einerseits steht zwar das politische Geschehen auf der Liste der medienbezogenen Themeninteressen der Deutschen ganz oben; für Informationen zu Politik werden aber vorrangig die klassischen Medien Fernsehen, Hörfunk, Tageszeitung genutzt. Das Internet wurde bei der Untersuchung zum „Informationsverhalten der Deutschen 2004“ (Blödorn et al. 2005: 643-644) von den Onlinern vorrangig für Freizeit- und Alltagsinformationen genutzt (z.B. Reisen, Musik, Finanzen). Auch eine direkte Frage nach dem Gewicht von politikbezogenen Inhalten an der gesamten Online-Nutzung im Rahmen einer Befragung aus dem Jahr 2003 in Deutschland zeigt, dass die Mehrheit der Menschen insgesamt nur einen recht kleinen Anteil ihrer Online-Zeit für politische Inhalte verwendet (Emmer \& Vowe 2004: 207). Tewksbury (2006) stellt hierzu in einer Untersuchung in den USA fest, dass sich die Zuwendung zu Online-Medien im Wahlkampf je nach Ereignisphase ändert - im Rahmen von ,Schlüssel-Events' mit größerem Informationsbedarf spielten Online-Quellen für die Befragten eine größere Rolle als in Phasen normaler, durchschnittlicher Wahlkampfkommunikation. Trotzdem ist die Rezeption von Nachrichten und anderen politischen Informationsangeboten auch in politischen ,Normalzeiten' ein fester Bestandteil der individuellen Internet-Nutzung vieler Onliner. Für immerhin ein gutes Drittel aller Deut- 
schen ist das Internet eine relevante Quelle politischer Informationen (siehe Tabelle 1). Der Unterschied zur Nutzung politischer Hintergrundinformationen im Fernsehen oder in PrintProdukten ist zwar weiterhin vorhanden, aber er ist nicht sehr groß: Ungefähr die Hälfte der Deutschen nutzt diese herkömmlichen Angebote regelmäßig (über Fernsehnachrichten hinaus, die von praktisch jedem genutzt werden). Außerdem zeigt sich, dass der durch das Netz erleichterte Zugang zu ungefilterten Informationen politischer Akteure (Politiker, Parteien) und alternativer Informationsanbieter (Weblogs) bemerkenswerte Reichweiten hat.

Tabelle 1: Nutzung politischer Online-Informationen (Anteil der deutschen Bevölkerung die dies mindestens "gelegentlich" im letzten Jahr gemacht hat)

\begin{tabular}{lccc}
\hline & $\mathbf{2 0 0 2}$ & $\mathbf{2 0 0 5}$ & $\mathbf{2 0 0 8}$ \\
\hline Politische Informationen im Internet & $27,3 \%$ & $39,1 \%$ & $38,2 \%$ \\
Politiker-Websites & $10,8 \%$ & $15,2 \%$ & $17,7 \%$ \\
Weblogs mit politischem Inhalt & - & - & $28,0 \%$ \\
\hline
\end{tabular}

Quelle: Emmer \& Wolling (2009: 98-99)

Für die USA ermitteln Smith \& Rainie (2008: 3) ähnliche Nutzerzahlen für politische OnlineInformationen wie in Deutschland: Kurz vor den US-Wahlen 2002 bezogen 23 Prozent der Amerikaner Informationen zur Wahl aus dem Netz, im Herbst 2004 waren es 34 Prozent, im Frühjahr 2008 bereits 40 Prozent. Ähnlich auch die Ergebnisse für Großbritannien, wo 200746 Prozent der Bürger angaben, Nachrichten im Internet zu lesen (Dutton \& Helsper 2007: 5; 67). Die relativ einheitlichen Ergebnisse in den drei westlichen Demokratien dürfen aber nicht darüber hinwegtäuschen, dass solche Erkenntnisse nur für mehr oder weniger pluralistischdemokratisch verfasste Gesellschaften verallgemeinerbar sind: In autoritären Regimen und Diktaturen ist der Umgang mit dem Internet geprägt von Verbots-, Überwachungs- und Propagandamaßnahmen der Regierenden (z.B. anlässlich der Oppositionsproteste in Myanmar 2007 oder im Iran 2009). Transparenz und Bürgerbeteiligung sind hier keine relevanten politischen Kategorien (Biedermann 1999; Domes 1996).

Das Netz als politische Informationsquelle besteht aus Milliarden Webseiten und anderen Informationsquellen (Datenbanken, Chats, Twitter etc.), über deren detaillierte Nutzung - nicht zuletzt wegen erheblicher methodischer Probleme etwa bei der Bestimmung von Grundgesamtheiten (z.B. Eingrenzung zu untersuchender Angebote) und der Stichprobenziehung - nur wenige gesicherte Daten vorliegen (Quandt 2008). Einerseits wird darauf hingewiesen, dass das Internet, anders als die alten Massenmedien, einen sog. ,Long-Tail' (Anderson 2008) an kleinen, individuellen Angeboten möglich macht, die sich im Einzelfall durchaus zu relevanten Orten öffentlicher Kommunikation entwickeln können. Andererseits zeigen die extrem hohen Besucherzahlen einiger weniger etablierter Informationsanbieter in Deutschland - Spiegel.de als Marktführer mit 322 Mio. Besuchen im ersten Quartal 2009, mit deutlichem Abstand zu Bild.de mit 190 Mio. auf Platz 2 (BITKOM 2009) - dass die Informationsströme im Internet, anders als der Vielfalts-Mythos des Internets vermuten lässt, stark zentralisiert und kanalisiert verlaufen. Dies 
lässt hinsichtlich möglicher gesellschaftlicher Fragmentierung, Agenda-Setting-Prozessen etc. nur begrenzte Veränderungen erwarten (mehr dazu weiter unten).

\subsection{Online-Medien als Gelegenheitsstruktur für politische Interaktionen}

Das interaktive Potenzial des Netzes gehört zu dessen meistdiskutierten Vorteilen gegenüber herkömmlichen Massenmedien. Über E-Mail, in Online-Foren, Blogs, Chats etc. können Bürger in verschiedenen Modi - zeitgleich oder asynchron, mehr oder weniger multimedial etc. - miteinander kommunizieren, wobei auch hier die Politik für den Einzelnen nur eine begrenzte Rolle spielt. Aktuelle Daten zeigen jedoch, dass hier ein großes Potenzial vorhanden ist, denn insbesondere die jüngste Generation der Online-Nutzer (14 bis 19 Jahre) nutzt diese interaktiven Angebote äußerst intensiv: Chats, Instant-Messaging und Online-Communities werden mittlerweile in dieser jungen Gruppe von ähnlich vielen Onlinern genutzt (76 bis 80 Prozent) wie E-Mail Kommunikation (85 Prozent), die die Liste der meistgenutzten Online-Aktivitäten in der Regel anführt (van Eimeren \& Frees 2009: 339).

Für die interpersonale Kommunikation zwischen Bürgern über Politik ist das Netz jedoch noch nicht der bevorzugte Ort: Im Durchschnitt unterhält sich nur etwa jeder sechste Deutsche online mit anderen Personen über politische Dinge, wobei auch hier der Generationenunterschied erheblich ist, denn in den jüngsten Nutzergruppen (16 bis 29 Jahre) waren es im Jahr 2007 mit 49 Prozent bereits fast die Hälfte (Emmer \& Wolling 2009: 100). Aber nicht nur Diskussionen mit Mitbürgern sondern auch die Kontaktaufnahme mit Politikern wird durch das Netz technisch erleichtert. Allerdings wird dafür, trotz der vielfältigen elektronischen Kontaktmöglichkeiten wie E-Mail, Politiker-Chats oder von Dritten bereitgestellten Interaktionsportalen wie Abgeordnetenwatch.de, das Netz nur von wenigen Bürgern genutzt (2007: 5,4 \%; Emmer \& Wolling 2009: 102). Wie auch bei den herkömmlichen Kontaktformen (Bürgersprechstunde, Brief, Telefon) sind einer regelmäßigen, intensiven Interaktion aufgrund der im Verhältnis zu den Bürgern geringen Zahl an Politikern natürliche Grenzen gesetzt. Diese Interaktionsmittel lassen sich aber auch anders einsetzen: In den USA werden sie verstärkt als Werbeinstrument durch die etablierte Politik verwendet. Vor den Präsidentschaftswahlen 2008 gaben 23 Prozent der Wähler an, mindestens einmal die Woche per E-Mail zur Unterstützung eines Kandidaten aufgerufen worden zu sein (Smith \& Rainie 2008). Die direkte Kontaktaufnahme mit den Bürgern spielt in Deutschland sowohl online als auch offline eine wesentlich geringere Rolle. Eine Erklärung hierfür könnten Unterschiede im Wahlsystem sein: Anders als in den USA werden in Deutschland Parteipräferenzen der Wähler nicht im Wählerverzeichnis erfasst; dadurch sind die Streuverluste und damit die Kosten der Kommunikation in Deutschland deutlich größer. Darüber hinaus ist eine individualisierte Ansprache ohne die Mitarbeit einer großen Zahl freiwilliger Wahlhelfer kaum zu realisieren. Eine dafür notwendige breite Mobilisierung der Bürger ist anders als in den USA untypisch für die Situation in Deutschland, in der Kampagnen eher durch stark strukturierte Parteiapparate kontrolliert und organisiert werden. 
Das Alter bzw. der Geburtsjahrgang ist im Bereich der interpersonalen politischen OnlineKommunikation einer der wichtigsten moderierenden Faktoren, noch vor den ebenfalls wichtigen Variablen Bildung oder politisches Interesse (Emmer \& Wolling 2009: 100). Herkömmliche interpersonale Interaktionen lassen sich dagegen viel stärker durch politisches Interesse erklären. Außerdem zeigt sich, dass im Bereich der interpersonalen politischen Online-Kommunikation jede neue Alterskohorte in den letzten Jahren bereits von Anfang an intensivere Aktivitäten an den Tag gelegt hat als die jeweils vorhergehende. Dies spricht dafür, dass sich in Zukunft solche Kommunikationsformen weiter durchsetzen und für alle Gesellschaftsbereiche - und damit auch für die Politik - weiter an Bedeutung gewinnen werden.

\subsection{Online-Medien als Gelegenheitsstruktur für politische Partizipation}

Anders als klassische Massenmedien stellen Online-Medien vielfältige Optionen für eine direktere Beteiligung des Einzelnen am öffentlichen Diskurs bereit: Ein wesentlicher Aspekt ist hier der sog. Bürgerjournalismus (siehe hierzu den Beitrag von Neuberger \& Quandt in diesem Band). Neben der Möglichkeit, als Bürger selbst Informationen und Meinungen in den öffentlichen Diskurs einzubringen (z.B. über einen eigenen Weblog), eröffnet das Internet zahlreiche weitere Beteiligungsoptionen, wie etwa Online-Petitionen, Abstimmungen, etc. Hierbei ist zu unterscheiden, inwieweit Bürger selbst online aktiv werden und welche Bedeutung solchen Aktivitäten hinsichtlich der Stabilität des politischen Systems zugewiesen wird: Intensive politische Aktivität kann durchaus auch ein Indikator für Protest und Unzufriedenheit sein; insofern ist für eine normative Bewertung dieser Ergebnisse eine Einbeziehung auch der Einstellungsdimension (siehe nächster Abschnitt) erforderlich.

Der Einfluss des Internets wird auf dem Feld der Partizipation vor allem unter der Mobilisierungsperspektive diskutiert. Die ,positive' Grundannahme lautet hier, dass das Netz Bürger zur stärkeren Beteiligung mobilisieren kann (Norris 2000). Dem steht allerdings die Hypothese der sich auflösenden Bürgerschaft gegenüber, nach der die wachsende Bedeutung des Internets zu einer gesellschaftlichen Fragmentierung führt, und die Nutzerschaft sich in zahllose Nischen vereinzelt (Putnam 2000).

Ein Phänomen, das als Beleg für die partizipationsfördernde Kraft des Internets dienen kann, ist die enorme Mobilisierung von Unterstützung und Beteiligung durch Wähler, die die stark online-gestützte Präsidentschaftswahlkampagne von Barack Obama 2008 in den USA erreicht hat (Fliegauf \& Novy 2009). Diese Kampagne hat unter den Bürgern Erwartungen hinsichtlich eines sich grundsätzlich verändernden Politikstils geweckt (Smith 2008). Es ist abzuwarten, ob die Mobilisierung nur ein Wahlkampfphänomen war oder ob sie eine neue Phase intensiver politischer Partizipation in den USA einleiten wird. In Barack Obamas Wahlkampagne 2008 verbanden sich nicht nur Kommunikationsformen und Medien auf neuartige Weise miteinander, auch die Trennung zwischen Partizipation des Einzelnen und Kampagnenführung durch Politiker und Organisationen verschwamm zunehmend, als die Kampagnenführung mit Hilfe des Netzes Inhalte und Organisation ihrer Aktionen zumindest teilweise den Bürgern und Wählern überließ. 
Unter den Bedingungen der deutschen Parteiendemokratie sind solche Phänomene, wie sie in den USA beobachtet werden konnten (für Beispiele siehe Moorstedt 2008), nur schwer denkbar, zumindest zur Zeit noch.

Mittlerweile liegen aus verschiedenen Quellen Daten vor, die zeigen, welche Optionen partizipativen politischen Handelns im Internet von den Bürgern wie intensiv genutzt werden. In der öffentlichen und wissenschaftlichen Diskussion standen dabei zuletzt die Kommunikationsformen des ,Social Web' (vgl. Schmidt 2009) bzw. der sog. ,User-Generated Content' im Mittelpunkt.

Tabelle 2: Politische Partizipation online (Anteil der deutschen Bevölkerung, die dies im letzten Jahr mindestens einmal gemacht hat)

\begin{tabular}{lcccr}
\hline & $\mathbf{2 0 0 2}$ & $\mathbf{2 0 0 5}$ & \multicolumn{2}{c}{$\mathbf{2 0 0 8}$} \\
\hline & & & Alle & $\mathbf{1 4 - 2 9 ~ J .}$ \\
\hline Online-Leserbriefe & $12,0 \%$ & $19,6 \%$ & $7,5 \%$ & $13,2 \%$ \\
Online-Petitionen & $3,8 \%$ & $5,1 \%$ & $6,2 \%$ & $5,7 \%$ \\
Online-Spenden & - & - & $0,7 \%$ & $1,7 \%$ \\
Online-Mitarbeit in Organisationen & $1,0 \%$ & $1,4 \%$ & $5,7 \%$ & $3,5 \%$ \\
Eigene Homepage mit Politikinhalt & $0,2 \%$ & $0,6 \%$ & $0,6 \%$ & $2,6 \%$ \\
Produktion politischer Podcasts/Online-Videos & - & - & $0,6 \%$ & $1,7 \%$ \\
\hline
\end{tabular}

Quelle: Emmer \& Wolling (2009: 98f.)

Die vorliegenden empirischen Ergebnisse zeigen: Partizipationsaktivitäten sind Minderheitenaktivitäten, online ebenso wie offline. Vergleicht man verschiedene mögliche Optionen, so sind Online-Leserbriefe die am häufigsten genutzte Partizipationsform (Tabelle 2). Hier finden sich in etwa doppelt so viele Aktive wie bei den herkömmlichen Leserbrief-Schreibern (Emmer 2005: 129). Das verdeutlicht, dass hier das Mobilisierungspotenzial des Internets tatsächlich teilweise realisiert wurde. Bei anderen Aktivitäten, etwa den Petitionen bzw. Unterschriftensammlungen, sieht das Verhältnis umgekehrt aus: Daran nehmen in Deutschland offline deutlich mehr Menschen teil als online (ebd.). Gleiches gilt für weitere Partizipationstätigkeiten, bei denen auch online ein gewisser Aufwand nötig ist bzw. Kosten anfallen, wie etwa die Produktion und Pflege eigener öffentlicher Kommunikationsangebote durch Websites, Weblogs oder Podcasts: Dass der Aufwand gegenüber der Produktion einer herkömmlichen Zeitung oder Fernsehsendung wesentlich kostengünstiger und einfacher zu realisieren ist, ist für den Einzelnen offenbar nicht ausschlaggebend: Der relative Aufwand ist zweifellos geringer, der absolute Aufwand aber vermutlich immer noch deutlich zu hoch.

Entscheidend für die Gültigkeit der Mobilisierungsthese ist allerdings, dass die beobachteten Aktivitäten erstens nicht einfach nur ,alte' Partizipation substituieren, sondern tatsächlich einen Partizipationszuwachs darstellen, und dass dieser Zuwachs sich zweitens kausal auf das Internet bzw. Online-Nutzung und -Kommunikation zurückführen lässt. Auch diese zweite Bedingung ist bereits in Forschungsprojekten untersucht worden: Rice \& Katz (2004) liefern auf Basis von 
Daten aus den US-Wahlkämpfen 1996 und 2000 Belege dafür, dass das Internet eher mobilisiert als demobilisiert, ebenso Weber et al. (2003), die jedoch darauf hinweisen, dass eine selektive Mobilisierung bestimmter Bevölkerungsgruppen digitale Klüfte verbreitern kann (zu Digital Divide vgl. die Beiträge von Marr \& Zillien, Hartmann \& Krotz sowie Schweiger in diesem Band). Krueger stellt hierzu fest, dass sich durch das Netz aber durchaus auch bisher weniger politisch aktive Personen mobilisieren lassen (Krueger 2002). Ebenfalls aus den USA stammt eine Reihe von Studien, die sich mit einem etwas weiter gefassten, zivilgesellschaftlichen Partizipationsbegriff (Civic Participation in Erweiterung von Political Participation) beschäftigen. Diese Untersuchungen kommen zu ähnlichen Ergebnissen: Es lassen sich begrenzte, positive Einflüsse der Internet-Nutzung auf gesellschaftliche Beteiligung beobachten, die zum Teil komplementär zu herkömmlichen Aktivitäten stattfinden (Best \& Krueger 2005; Katz \& Rice 2005; Kluver 2005; Pearson 2005; Shah et al. 2002; Uslaner 2004). Es gibt allerdings auch einzelne kritische Ergebnisse: Nie \& Erbring (2002) stellten in einer frühen Studie fest, dass die online verbrachte Zeit in einer negativen Beziehung zur Qualität der allgemeinen (nicht politikbezogenen) sozialen Kontakte der Befragten stand.

Anders als die meisten dieser Studien, die mit Querschnittdaten arbeiten, nähern sich Jennings \& Zeitner (2003) der Wirkungsfrage mit Daten eines Panels von Highschool-Absolventen des Jahrgangs 1965 und ermittelten positive Effekte von politischer Online-Informationsnutzung. Für Deutschland liegen hierzu Daten aus einer mehrjährigen Panel-Untersuchung vor (Emmer 2005; Emmer \& Vowe 2004; Vowe et al. 2007), die eine begrenzte Mobilisierung der Deutschen zeigen: Die Anschaffung eines Internet-Zugangs intensivierte die Nutzung politischer Informationsangebote und teilweise auch der interpersonalen Kommunikation der Befragten über Politik. Es gab keine Hinweise auf eine Substituierung herkömmlicher politischer Kommunikation oder gar auf eine Abwendung von der Politik. Ein Einfluss auf politische Partizipationsaktivitäten konnte allerdings nicht nachgewiesen werden.

\section{Folgen des Internets für die öffentliche Kommunikation}

\subsection{Wirkung auf politische Themensetzung und öffentliche Meinung: Agenda Building, Agenda Setting, Schweigespirale}

Die Besonderheiten des Internets - der egalitäre Zugang, der geringere Einfluss journalistischer Gatekeeper sowie zeitlicher und räumlicher Beschränkungen - lassen erwarten, dass Thematisierungsprozesse hier anders verlaufen und dadurch die Themen-Agenda in InternetÖffentlichkeiten eine andere Gestalt hat als die klassische Medienagenda. Die abweichenden öffentlichen Diskurse und die damit einhergehende andere Themensetzung durch die Debatte könnte einen Einfluss auf die Wahrnehmung von Problemfeldern durch die InternetNutzer haben und sich auf deren Themen-Agenda auswirken. Die grundlegende Hypothese lautet, dass Online-Kommunikation das Thematisierungsmonopol der klassischen Massenmedien 
und mit ihnen die Macht von Journalisten und Medienunternehmen brechen kann (Baum \& Groeling 2008: 346). Dies kann beispielsweise die Chancen für kleinere, im klassischen Mediensystem benachteiligte Interessenvertretungen (NGOs, Bürgerinitiativen etc.) erhöhen, ihre Themen in die öffentliche Debatte einzubringen. Allerdings geht damit die Befürchtung einher, dass die größere Vielfalt an Kommunikatoren bei geringerem Einfluss von Gatekeepern zu einer Fragmentierung von Öffentlichkeit führen könne (Holtz-Bacha 1998; Sunstein 2001). Empirische Studien hierzu lassen sich grob danach unterscheiden, ob sie sich auf Basis von Inhaltsanalysen für Veränderungen des medialen Themenspektrums (Agenda Building und Intermedia Agenda Setting) interessieren, oder ob sie - in der Regel durch Einsatz von Methodenkombination - Wirkungen von Medienagenden auf Rezipienten (Agenda Setting) in den Blick nehmen.

Hinsichtlich des Agenda Building-Prozesses wurde bereits früh im Zusammenhang mit Wahlkämpfen (vor allem in den USA) untersucht, welchen Einfluss Kandidaten-Websites auf Medieninhalte haben. Eine Analyse des Bush-Gore-Wahlkampfs im Jahr 2000 zeigte moderate Effekte der Kandidaten-Websites auf die Themenagenden von Tageszeitungen und Fernsehnachrichten (Ku et al. 2003: 535-536), wobei der Einfluss der Kandidaten-Websites im Zeitverlauf deutlicher war als das Intermedia Agenda Setting durch die anderen Medien. Auch Webangebote von öffentlichen Verwaltungen können Einfluss sowohl auf die Medien- als auch auf die Publikumsagenda haben (Winsvold 2007). Dabei ist von besonderer Relevanz, dass OnlineAngebote bevorzugte Quellen von Journalisten sind und insbesondere von gebildeten Eliten und Meinungsführern genutzt werden (Woodly 2008). Eine Inhaltsanalyse von klassischen Medienangeboten und Weblogs im Kontext der US-Präsidentschaftswahlen 2004 ermittelte wenige Intermedia-Agenda-Setting-Effekte zwischen Online- und Offline-Angeboten (Jae Kook 2007). In einer Untersuchung zur Präsidentenwahl in Süd-Korea im Jahr 2000 untersuchten Byoungkwan et al. (2005) den Einfluss von Online-Medien auf die Berichterstattung klassischer Medienangebote und konnten einen Einfluss von Internet-Bulletin Boards auf die Medienberichterstattung ermitteln. Im Gegensatz dazu zeigte eine Untersuchung im Nahen Osten von Curtin \& Gaithner (2004), dass dialogorientierte englischsprachige Online-Angebote nur wenig zur medialen Themen-Agenda beitrugen.

Eine Untersuchung, die über die Prüfung der Agenda-Setting-Hypothese hinausgeht, ist die breit angelegte inhaltsanalytische Studie zum Thema Genfood von Rucht et al. (2008). Den Autoren ging es darum, Akteure, Kommunikationsstrukturen und Inhalte (Umfang, Themen, Argumente, Verknüpfungen) der Debatte in Zeitungen und Online-Medien im Vergleich zu untersuchen und daraus Schlüsse auf die Veränderung von öffentlichem Diskurs und Öffentlichkeit zu ziehen. Im Ergebnis zeigen sich auch diese Autoren überrascht über den geringen Unterschied zwischen herkömmlich-medialem und Online-Diskurs (Rucht et al. 2008: 121), auch wenn sich im Detail interessante Unterschiede offenbarten (ähnliche Befunde auch bei Gerhards \& Schäfer 2007; Schweiger \& Weihermüller 2008).

Um die Folgen der Online-Kommunikation für Agenda-Setting-Prozesse zu untersuchen, beschäftigten sich Dalrymple und Scheufele (2007) mit dem Wissen, das Personen über Internet- und traditionelle Nachrichtenquellen erhalten und stellten fest, dass sich Online-Nutzer ein 
vertieftes Wissen über die Themen auf der medialen Agenda aneignen und damit OnlineKommunikation, die vom Nutzer mehr Eigeninitiative verlangt, inhaltlich stärkere Spuren hinterlassen kann als herkömmliche Medien.

Eine Untersuchung von Schönbach et al. (2005: 246f.) in den Niederlanden geht der Hypothese nach, dass Onliner eine engere Themen-Agenda hätten: Zeitungsleser bekämen demnach einen breiteren Überblick über relevante Themen, während Internet-Nutzer aufgrund ihrer stärker fokussierten Nutzung eine weniger Themen umfassende Agenda hätten. Grundsätzlich wird diese Annahme durch die Ergebnisse unterstützt, wobei höhere Bildung bei Online-Nutzern die Unterschiede zwischen den Gruppen verschwinden lässt.

Fragt man nach einem möglichen moderierenden Einfluss des Internets auf Agenda-SettingWirkungen von herkömmlichen Medien wie Fernsehen oder Tageszeitung, so liegt die Vermutung nahe, dass jüngere Altersgruppen durch ihre intensivere Online-Nutzung weniger stark von der Themen-Agenda klassischer Medien beeinflusst werden. Coleman \& McCombs (2007: 502) fanden in einer US-Studie zwar leicht schwächere Effekte herkömmlicher Medienangebote auf die jüngeren Mediennutzer; insgesamt zeigten sich jedoch über alle Altersgruppen hinweg relativ stabil die gleichen Themenagenden, was für einen bisher nur geringen fragmentierenden Einfluss des Netzes spricht. Ähnliches ermittelten für Deutschland Emmer \& Wolling (2007): Auch hier zeigten sich keine dramatischen Unterschiede, allerdings ließen sich die Differenzen in der Themennennung zwischen Onlinern und Offlinern durchaus im Sinne von internetbezogenen Hypothesen interpretieren. So nannten Online-Nutzer häufiger globale und seltener regionale Themen als Offliner. Für die Bestätigung einer Fragmentierungshypothese durch ein höheres $\mathrm{Ma} ß$ an Vielfalt der genannten Themen bei den Onlinern fanden sich jedoch keine Belege. Ebenfalls keine Hinweise für eine Fragmentierung fanden Tewksbury \& Althaus (2000). Vielmehr zeigte sich, dass die Nutzung der Online-Angebote den Rezipienten offenbar inhaltlich schmalere Themenagenden vermittelt. Allerdings basiert ihre Studie auf einer experimentellen Untersuchung, die sich mit den psychologischen Verarbeitungsmechanismen bei der Nutzung von sequenziell auf Papier dargebotenen Informationen im Vergleich zu hypermedialen elektronischen Informationen beschäftigte und nicht mit dem Einfluss des veränderten Medienangebots generell. Rußmann (2007) liefert mit einem qualitativen Ansatz einige Hinweise darauf, dass selbst bei Intensivnutzern des Internets die Online-Kommunikation die Nutzung klassischer Informationsangebote wie Fernsehen, Radio oder Zeitung nicht vollständig ablöst, sondern eher komplementär erfolgt, was mögliche Wirkungen der Online-Angebote auf die Publikumsagenda vermutlich abschwächt.

Auch auf gesellschaftliche Meinungsbildungsprozesse, insbesondere auf SchweigespiralEffekte sind Einflüsse durch das Internet zu erwarten: Die verteilte Struktur und die größere Anonymität computervermittelter Kommunikation sollte Menschen eine freiere Äußerung auch abweichender Meinungen erlauben und Konformitätsdruck abmildern (Turkle 1999: 645; vgl. auch den Beitrag von Döring in diesem Band). Allerdings ist der Forschungsstand hierzu begrenzt. Erste empirische Untersuchungen zeigen, dass die Vermutung tatsächlich zutreffen kann: In einer Studie zur Diskussion und Meinungsäußerung über Homosexualität in den USA zeigte 
sich, dass die Untersuchungspersonen eher äußerungsbereit waren, wenn sie sich statt einer Face-to-Face-Situation eine Chat-Room-Diskussion vorstellten (Ho \& McLeod 2008). Waymer (2007) argumentiert auf Basis von Fallstudien, dass das Internet Minderheiten bzw. kleineren Interessengruppen eine stärkere Stimme im öffentlichen Diskurs geben kann. Für Deutschland zeigt Mayer-Uellner (2003) in einer Analyse universitärer Diskussionsforen, dass die konkrete Ausgestaltung der Online-Kommunikation hinsichtlich der Anonymität der Diskussionspartner einen Einfluss auf die Äußerungsbereitschaft hat: im Allgemeinen zeigten sich dann stärkere Schweigespiral-Effekte, wenn die Gesprächspartner identifizierbar waren. Roessing (2008) untersuchte am Beispiel einer Inhaltsanalyse von Wikipedia, welche komplexen Strukturen von Kommunikation (es wurden Meinungsbildungsprozesse auf Inhalts-, Artikel- und Metadiskussionsebene identifiziert) sich in einem solchen dynamischen Umfeld ergeben können, wobei aufgrund fehlender Befragungsdaten eine direkte Beobachtung von Schweigespiral-Effekten nicht möglich war.

\subsection{Wirkungen auf politische Einstellungen: Framing, Priming, Persuasion}

Sowohl aus Perspektive strategischer Kommunikation wie aus demokratietheoretischer Perspektive ist die Frage relevant, inwieweit Einstellungen zu politischen Programmen, Parteien, Kandidaten und zum politischen System durch die Strukturen der Online-Kommunikation verändert werden.

Hinsichtlich des Einflusses des Internets auf die Wahrnehmung von Demokratie (z.B. auf Demokratievertrauen bzw. -verdrossenheit) und auf allgemeine Einstellungen zur Politik - Vertrauen in Mitbürger, politische Einflussüberzeugung, Responsivität des politischen Systems, etc. - herrschen Hypothesen vor, die positive Effekte erwarten lassen (Polat 2005). Die bisherigen Befunde zur Wirkung herkömmlicher Medien (Fernsehen/Zeitung) zeigen hingegen keine eindeutige Tendenz, sondern deuten auf differenzielle Wirkungen: Die Nutzung bestimmter Medien(inhalte) führt zur Entfremdung und zu negativeren Einstellungen gegenüber politischen Institutionen und dem politischen Prozess (Mediamalaise), während andere Medien(inhalte) eher positive Wirkungen zeitigen (Holtz-Bacha 1990). Mittlerweile wurden aber auch einige Studien durchgeführt, in denen die Wirkung der Internet-Nutzung auf Einstellungen zur Politik geprüft wurde (u.a. Norris 2000, Wolling 2009). Insgesamt erweist sich der Forschungsstand als uneinheitlich, was aber angesichts der verschiedenen Einstellungsindikatoren und unterschiedlicher Messungen der Internet-Nutzung kaum überraschen kann. Hinzu kommt, dass ein Großteil der vorliegenden Studien auf Querschnittdaten beruht, und Schlüsse auf Wirkungen des Internets dabei nur durch Gruppenvergleiche unter Kontrolle soziodemographischer Variablen gezogen werden können. Inhaltsanalysen, die das entsprechende Wirkungspotenzial der InternetAngebote bestimmen, fehlen vollständig - nicht zuletzt wegen kaum lösbarer methodischer Probleme.

Bislang sind empirische Studien vor allem im Kontext von Online-Wahlkämpfen und -Kampagnen entstanden. In einer Untersuchung der US-Präsidentschaftswahlkämpfe 1996 und 
2000 ermittelten Johnson \& Kaye (2003), dass die befragten Bürger nicht nur angaben, das Netz habe ihre politische Aktivität intensiviert, sondern ihre Datenanalysen zeigten auch, dass politisch interessierte Internet-Nutzer insgesamt ihren politischen Einfluss auf die Politik als größer einschätzen. In Korea, wo demokratische Politik erst eine relativ kurze Geschichte hat, wird den Online-Medien eine bedeutende Rolle bei der Veränderung der politischen Kommunikation zugeschrieben. Hierzu gibt es eine ganze Reihe von Studien. Kim \& Johnson (2006: 13) berichten z.B. von positiveren Einstellungen gegenüber dem politischen System bei intensiven OnlineNutzern in Korea, ein Ergebnis, das allerdings durch die besondere politische Situation und das Misstrauen der Koreaner gegenüber traditionellen Medien beeinflusst sein könnte.

Nisbet \& Scheufele (2004) konnten ebenfalls positive Effekte des Internet-Zugangs und der Nutzung von Kampagneninformationen im Internet auf politische Einflussüberzeugung (political efficacy) feststellen. Ähnliche Befunde erzielten Kenski und Stroud (2006), die zu dem Ergebnis kamen, dass sowohl die Einflussüberzeugung (internal efficacy) als auch die Einschätzung der Responsivität der Politiker (external efficacy) bei Personen mit Internet-Zugang etwas höher ausfallen. Andere Ergebnisse ermittelte hingegen Muhlberger (2003), der in einer Querschnittstudie die politischen Einstellungen von Bürgern verglich, die online und offline aktiv waren und dabei zu dem Schluss kam, dass diese sich hinsichtlich ihres Aktivitätsniveaus und auch hinsichtlich politischer Einstellungen nicht signifikant unterschieden.

Wang (2007) prüfte in einem komplexen Analysemodell die Interaktion zwischen Wertorientierungen, Einstellungen und politischer Internet-Nutzung, denn Einstellungen können sowohl Ursache wie Folge politischer Online-Nutzung sein. Die Ergebnisse zeigen, dass ein Modell, das Online-Nutzung als Ursache für steigendes Vertrauen und Einflussüberzeugung betrachtet, eine größere Erklärungskraft aufweist als das Konkurrenzmodell mit anderer Wirkungsrichtung, bei dem diese Einstellungen als Prädiktoren für Online-Nutzung geprüft wurden. Wolling (2009) prüfte für Deutschland die Mediamalaise-These unter Berücksichtigung von Online-Kommunikation und mit Hilfe eines Paneldesigns. Es zeigten sich in keinem der Modelle negative Effekte der Internet-Nutzung auf politische Einstellungen, sondern im Gegenteil eine Verbesserung der Werte für generalisiertes soziales Vertrauen, Demokratiezufriedenheit und Einflussüberzeugung. Die Analyse lässt dabei den Schluss zu, dass die Wirkungsrichtung in den meisten Fällen nicht einseitig ist, sondern sich Nutzung und Einstellungen gegenseitig stabilisieren.

Neben Veränderungen von allgemeinen politischen Einstellungen stellt sich jedoch auch die Frage, ob Online-Kommunikation einen Einfluss auf die Einschätzung von konkreten Themen, Kandidaten oder Organisationen hat: Es ist anzunehmen, dass sowohl die inhaltlichen Veränderungen des Medienangebots (Meinungsvielfalt durch Blogs, Probleme mit der Glaubwürdigkeit von Online-Quellen etc.) als auch die Art und Weise der Inhaltsvermittlung (hypertextuell, multimodal) Auswirkungen auf die Wahrnehmung von Inhalten hat und dies wiederum Einfluss auf die Bewertung von Themen, Kandidaten und Parteien sowie anderen Organisationen nimmt.

In einer Analyse der im Internet stattfindenden Debatte über das Rauchverbot in Gaststätten, die die Kommunikate von politischen Akteuren, klassischen Medienanbietern und auch Privatpersonen einschloss, ermittelten Schweiger \& Weihermüller (2008) nur geringe Unterschiede 
zwischen den inhaltlichen Schwerpunkten der Online-Debatte und den aus einer Bevölkerungsbefragung gewonnen Ansichten von Bürgern. Fridkin et al. (2008) liefern ein Beispiel für eine Studie, in der als unabhängige Variable zur Erklärung des positiven ,Spins' der USFernsehdebatten 2004 nicht nur Fernsehen und Zeitung, sondern auch Online-Quellen verwendet wurden, wobei der Vergleich von Online- und herkömmlichen Quellen nicht im Fokus der Untersuchung stand. Insgesamt zeigte sich, dass die Rezeption von Wahlkampfkommentaren unabhängig davon in welchen Medien - zumindest kurzfristig die Kandidatenbewertungen der Bürger beeinflusste.

Wall (2006) untersuchte die Frage, wie der Golfkrieg in Weblogs diskutiert wurde, wobei er eher konventionelle Frames wiederfand, die bereits durch herkömmliche Massenmedien geprägt waren. Ähnliches zeigten Gerhards \& Schäfer (2007) beim Thema „Humangenomforschung“ für Deutschland: Sie fanden bei einem Vergleich von Tageszeitungen und Suchmaschinenergebnissen im Internet keine wesentlichen Unterschiede im Framing des Themas. Auch Zhou \& Moy (2007) interessierten sich für die Interaktion von klassischen und Online-Medien bei der inhaltlichen Konstruktion von öffentlichen Diskursen. In ihrer Untersuchung prüften sie, wie Weblog-Diskussionen und Medienberichterstattung über ein Unfall-Ereignis in China sich gegenseitig beeinflussen. Für eine frühe Phase einer Themenkarriere konnten sie einen Einfluss von Weblogs auf klassische Medien feststellen, im weiteren Verlauf dominierte dann die Themendefinition durch die herkömmlichen Medienangebote.

Eine Untersuchung von politischer bzw. öffentlicher Kommunikation im Internet auf sprachlicher Ebene (Warnick 2007) betont den grundsätzlich neuen Umgang mit Texten und Argumentationslinien in Online-Umgebungen: Die eher lineare Logik klassischer Argumentation ist in einem hypertextuellen Umfeld nicht mehr richtig abbildbar, hier spielen die individuellen Konstruktionsleistungen der Rezipienten eine größere Rolle. In einer Reihe von Fallstudien (z.B. MoveOn.org oder Georgewbush.org) zeigt die Autorin, wie politische Akteure durch eine gezielte Kombination medialer Elemente und Verknüpfungen ihre Positionen argumentativ im Online-Umfeld kommunizieren.

\section{$5 \quad$ Fazit}

Der Online-Kommunikation wurde und wird eine Schlüsselrolle bei der Transformation politischer Öffentlichkeit zugeschrieben. Neben demokratietheoretisch begründeten Hoffnungen auf mehr Partizipation der Bürger am öffentlichen Diskurs durch das Netz stehen Befürchtungen hinsichtlich eines Funktionsverlusts der Medien als Foren politischer Öffentlichkeit, der durch die Online-Medien nicht kompensiert oder sogar noch verschärft werden könnte. Der in diesem Beitrag vorgelegte Überblick über den aktuellen Forschungsstand zeigt erstens, dass nur wenige der vermuteten negativen Effekte bisher tatsächlich eingetreten sind: Der Zutritt zum Internet hat nicht zu einer Abwendung von gesellschaftlich-politisch relevanten Handlungsweisen, wie der Rezeption politischer Informationen oder von politischer und gesellschaftlicher Partizipation 
geführt. Auch zeigen sich keine Erosionstendenzen in der politischen Kultur, etwa bei den Einstellungen zu Politik oder Demokratie. Es finden sich im Gegenteil eher Hinweise auf positive Entwicklungen bei der Beteiligung am öffentlichen Diskurs oder auch grundsätzlich positive Veränderungen der Einstellungen der Bürger zum demokratischen politischen System.

Allerdings sind diese positiven Effekte limitiert - von einer neuen athenischen Demokratie, wie sie Auguren des Netzes wie Al Gore Anfang der 1990er-Jahre erwartet haben, ist 15 Jahre später noch wenig zu sehen. Dies kann einerseits in grundsätzlichen Strukturbedingungen moderner Demokratien begründet sein, die sich nicht so einfach durch neue Medien- und Kommunikationstechniken beseitigen lassen, etwa das quantitative Ungleichgewicht von Entscheidungsträgern und Bürgern als Souverän in repräsentativ-demokratischen Systemen. Eine andere Ursache hierfür könnte die zunehmende Komplexität der Gesellschaft und ihrer Regeln sowie die wachsende Unübersichtlichkeit der Probleme und Lösungsoptionen sein. Andererseits ist aber auch denkbar, dass die technische Realisierung der Online-Kommunikation mit der Entwicklung der gesellschaftlichen Erwartungen nicht Schritt gehalten hat. Die Debatte um das sog. Web 2.0 oder Social Web mag dafür ein Beleg sein: Der Begriff beschreibt im Prinzip nichts Neues, sondern nur die schon immer im Internet angelegten, bisher aber z.B. wegen Bandbreiten- oder anderen Restriktionen technisch noch nicht realisierbaren Kommunikationseigenschaften, wie die Multimedialität der Online-Inhalte (Podcasts, Videostreams etc.), die Rolle von User-Generated Content, Community Building und anderes mehr.

Dritte wesentliche Schlussfolgerung ist, dass die Veränderung der öffentlichen politischen Kommunikation durch das Internet ein noch laufender Prozess ist, dessen Ende nicht absehbar ist. Es spricht wenig dafür, dass mit einer annähernden Vollabdeckung der Bevölkerung (oder zumindest wesentlicher Teile davon) mit Online-Zugängen sich ein neues technisches Medium etabliert hat und fortan in einer gewissen Form dauerhaft seine Rolle spielt. Vielmehr ist OnlineKommunikation durch eine erhebliche Dynamik sowohl in der technischen Entwicklung wie auch der sozialen und kulturellen Aneignung gekennzeichnet, die in wesentlichen Generationenbrüchen bei der Nutzung von Medien ihren Ausdruck findet (Emmer et al. 2006; Schmidt et al. 2009: 6) - und das auch im politischen Kontext (vgl. auch Hartmann \& Krotz in diesem Band).

Infolge dieser Dynamik nimmt der Forschungsbedarf hinsichtlich zukünftiger Entwicklungen der politischen Online-Kommunikation eher noch zu als ab, da weitere Transformationen der politischen Öffentlichkeit zu erwarten sind. Aber auch der Überblick über den Forschungsstand zu den klassischen politischen Mediennutzungs- und Wirkungsmustern online und offline zeigt, dass viele Fragen, etwa zum Agenda Setting oder zur persuasiven Online-Kommunikation, erst ansatzweise beantwortet sind. Da viele Studien hierzu nur explorativen oder Fallstudiencharakter haben, ist die Herausforderung hier nicht zuletzt eine methodische: Es gilt, die Qualität und damit die Aussagekraft der Forschung durch Verknüpfung von Datenquellen und von methodischen Ansätzen sowie insbesondere durch elaborierte, den jeweiligen Forschungsproblemen angemessene Untersuchungsdesigns (im Bereich der Wirkungsforschung v.a. experimentelle oder Paneluntersuchungen mit Mehrfachmessung) zu verbessern. 


\section{Literaturtipps}

Emmer, M. (2005). Politische Mobilisierung durch das Internet? München: Reinhard Fischer.

Moorstedt, T. (2008). Jeffersons Erben. Wie die digitalen Medien die Politik verändern. Frankfurt am Main: Suhrkamp.

Marcinkowski, F. \& Pfetsch, B. (Hrsg.) (2009). Politik in der Mediendemokratie. Politische Vierteljahresschrift, Sonderheft 42. Wiesbaden: VS.

\section{Literatur}

Anderson, C. (2008). The Long Tail: Why the Future of Business is Selling Less of More. New York: Hyperion.

Baum, M. A. \& Groeling, T. (2008). New Media and the Polarization of American Political Discourse. Political Communication, 25(4): 345-365.

Beck, K. (2006). Computervermittelte Kommunikation im Internet. München: Oldenbourg.

Best, S. J. \& Krueger, B. S. (2005). Analyzing the Representativeness of Internet Political Participation. Political Behavior, 27(2): 183-216.

Biedermann, T. (1999). Internetanwendung in China: Die vier 'Nein': Dissidenten, Pornographie, Tibet oder Taiwan. Online: http://www.politik-digital.de/text/netzpolitik/china/ index.shtml (04.12.1999).

BITKOM (2009). Nachrichtenseiten im Internet legen weiter zu. Online: http://www.bitkom .org/de/ presse/8477_58841.aspx (18.03.2010).

Blödorn, S., Gerhards, M. \& Klingler, W. (2005). Informationsnutzung und Medienauswahl. Media Perspektiven, Heft 12: 638-646.

Brants, K. (2005). Guest Editor's Introduction: The Internet and the Public Sphere. Political Communication, 22(2): 143-146.

Brecht, B. (1975). Der Rundfunk als Kommunikationsapparat. Rede über die Funktion des Rundfunks. In Brecht, B. (Hrsg.), Gesammelte Werke. Band 18: Schriften zur Literatur und Kunst 1 (4. Auflage, S. 127-134). Frankfurt am Main: Suhrkamp.

Browning, G. (1995). Electronic Democracy. Using the Internet to Influence American Politics. Wilton: Pemberton Press.

Byoungkwan, L., Lancendorfer, K. \& Ki Jung, L. (2005). Agenda-Setting and the Internet: The Intermedia Influence of Internet Bulletin Boards on Newspaper Coverage of the 2000 General Election in South Korea. Asian Journal of Communication, 15(1): 57-71.

Coleman, R. \& McCombs, M. (2007). The Young and Agenda-Less: Exploring Age-Related Differences in Agenda Setting on Generation X, Baby Boomers, and the Civic Generation. Journalism \& Mass Communication Quarterly, 84(3): 495-508.

Curtin, P. A. \& Gaither, T. K. (2004). International Agenda-Building in Cyberspace: A Study of Middle East Government English-Language Websites. Public Relations Review, 30(1): 2536.

Dahlberg, L. (2001). Computer-Mediated Communication and the Public Sphere: A Critical Analysis. Journal of Computer Mediated Communication, 7(1). Online: http://jcmc.indiana.edu/vol7/issue1/dahlberg.html (18.03.2010). 
Dalrymple, K. E. \& Scheufele, D. A. (2007). Finally Informing the Electorate? How the Internet Got People Thinking About Presidential Politics in 2004. Harvard International Journal of Press/Politics, 12(3): 96-111.

Domes, J. (1996). Diktaturen im Zeitalter der neuen Medien. Bertelsmann Briefe, 135: 14-16.

Dutton, W. H. \& Helsper, E. J. (2007). The Internet in Britain: 2007. Oxford: Oxford Internet Institute.

van Eimeren, B. \& Frees, B. (2009). Ergebnisse der ARD/ZDF-Online-Studie 2009: Der Internetnutzer 2009 - multimedial und total vernetzt? Media Perspektiven, Heft 7: 334-348.

Emmer, M. (2005). Politische Mobilisierung durch das Internet? Eine kommunikationswissenschaftliche Untersuchung zur Wirkung eines neuen Mediums. München: Reinhard Fischer.

Emmer, M., Füting, A. \& Vowe, G. (2006). Wer kommuniziert wie über politische Themen? Eine empirisch basierte Typologie individueller politischer Kommunikation. Medien \& Kommunikationswissenschaft, 54(2): 216-236.

Emmer, M. \& Vowe, G. (2004). Mobilisierung durch das Internet? Ergebnisse einer empirischen Längsschnittuntersuchung zum Einfluss des Internets auf die politische Kommunikation der Bürger. Politische Vierteljahresschrift, 45(2): 191-211.

Emmer, M. \& Wolling, J. (2007). Leben in verschiedenen Welten? Themenagenden von Offlinern und Onlinern im Vergleich. In Kimpeler, S., Mangold, M. \& Schweiger, W. (Hrsg.), Die digitale Herausforderung. Zehn Jahre Forschung zur computervermittelten Kommunikation (S. 239-250). Wiesbaden: VS.

Emmer, M. \& Wolling, J. (2009). 'Online Citizenship'? Die Entwicklung der individuellen politischen Beteiligung im Internet. In Bertelsmann Stiftung (Hrsg.), Lernen von Obama? Das Internet als Ressource und Risiko von Polittk (S. 83-116). Gütersloh: Verlag Bertelsmann Stiftung.

Fliegauf, M. T. \& Novy, L. (2009). "E-bama" - Amerikas erster "Internetpräsident" und die Rolle des World Wide Web für moderne politische Führung. In Bertelsmann Stiftung (Hrsg.), Lernen von Obama? Das Internet als Ressource und Risiko für die Politik (S. 185199). Gütersloh: Verlag Bertelsmann Stiftung.

Fridkin, K. L., Kenney, P. J., Gershon, S. A. \& Woodall, G. S. (2008). Spinning Debates: The Impact of the News Media's Coverage of the Final 2004 Presidential Debate. Harvard International Journal of Press-Politics, 13(1): 29-51.

Gerhards, J. (1995). Politische Öffentlichkeit. Ein system- und akteurstheoretischer Bestimmungsversuch. Kölner Zeitschrift für Soziologie und Sozialpsychologie, 47(1): 77-105.

Gerhards, J. (1998). Öffentlichkeit. In Jarren, O., Sarcinelli, U. \& Saxer, U. (Hrsg.), Politische Kommunikation in der demokratischen Gesellschaft. Ein Handbuch (S. 268-274). Opladen, Wiesbaden: Westdeutscher Verlag.

Gerhards, J. \& Neidhardt, F. (1993). Strukturen und Funktionen moderner Öffentlichkeit. Fragestellungen und Ansätze. In Langenbucher, W. R. (Hrsg.), Politische Kommunikation: Grundlagen, Strukturen, Prozesse (S. 52-89). Wien: Braumüller.

Gerhards, J. \& Schäfer, M. S. (2007). Demokratische Internet-Öffentlichkeit? Ein Vergleich der öffentlichen Kommunikation im Internet und in den Printmedien am Beispiel der Humangenomforschung. Publizistik, 52(2): 210-228.

Gore, A. (1994). Rede vor der World Telecommunication Development Conference, Buenos Aires, 21.03.1994. Online: http://www.itu.int/itudoc/itu-d/wtdc/wtdc1994/speech/gore.html (18.03.2010). 
Guggenberger, B. (1995). Demokratie/Demokratietheorie. In Nohlen, D. \& Schultze, R.-O. (Hrsg.), Lexikon der Politik. Band I: Politische Theorien (S. 36-49). München: C.H. Beck.

Habermas, J. (1962). Strukturwandel der Öffentlichkeit. Untersuchungen zu einer Kategorie der bürgerlichen Gesellschaft. Neuwied, Berlin: Luchterhand.

Habermas, J. (1991). Strukturwandel der Öffentlichkeit. Frankfurt am Main: Suhrkamp.

Ho, S. S. \& McLeod, D. M. (2008). Social-Psychological Influences on Opinion Expression in Face-to-Face and Computer-Mediated Communication. Communication Research, 35(2): 190-207.

Höflich, J. R. (1997). Zwischen massenmedialer und technischer Kommunikation. Der Computer als Hybridmedium und was die Menschen damit machen. In Beck, K. \& Vowe, G. (Hrsg.), Computernetze - ein Medium öffentlicher Kommunikation?(S. 85-104). Berlin: Spiess.

Holtz-Bacha, C. (1990). Videomalaise Revisited: Media Exposure and Political Alienation in West Germany. European Journal of Communication, 5(1): 73-85.

Holtz-Bacha, C. (1998). Fragmentierung der Gesellschaft durch das Internet? In Gellner, W. \& von Korff, F. (Hrsg.), Demokratie im Internet (S. 219-226). Baden-Baden: Nomos.

Imhof, K. (2003). Öffentlichkeitstheorien. In Bentele, G., Brosius, H.-B. \& Jarren, O. (Hrsg.), Öffentliche Kommunikation. Handbuch Kommunikations- und Medienwissenschaft (S. 193209). Wiesbaden.

Jae Kook, L. (2007). The Effect of the Internet on Homogeneity of the Media Agenda: A Test of the Fragmentation Thesis. Journalism \& Mass Communication Quarterly, 84(4): 745-760.

Jennings, K. M. \& Zeitner, V. (2003). Internet Use and Civic Engagement: A Longitudinal Analysis. Public Opinion Quarterly, 67(3): 311-334.

Katz, J. E. \& Rice, R. E. (2005). Syntopia: Access, Civic Involvement, and Social Interaction on the Net. In Wellman, B. \& Haythornthwaite, C. (Hrsg.), The Internet in Everyday Life (S. 114-138). Malden, Oxford, Carlton: Blackwell.

Kenski, K. \& Stroud, N. J. (2006). Connections Between Internet Use and Political Efficacy, Knowledge, and Participation. Journal of Broadcasting \& Electronic Media, 50(2): 173-192.

Kim, D. \& Johnson, T. J. (2006). A Victory of the Internet over the Mass Media? Examining the Effects of Online Media on Political Attitudes in South Korea. Asian Journal of Communication, 16(1): 1-18.

Kluver, R. (2005). Political Culture in Online Politics. In Consalvo, M. \& Allen, M. (Hrsg.), Internet Research Annual: Selected Papers From the Association of Internet Researchers Conference 2003, Volume 2 (S. 75-93). New York: Peter Lang.

Krueger, B. S. (2002). Assessing the Potential of Internet Political Participation in the United States - A Resource Approach. American Politics Research, 30(5): 476-498.

Ku, G., Kaid, L. L. \& Pfau, M. (2003). The Impact of Web Site Campaigning on Traditional News Media and Public Information Processing. Journalism \& Mass Communication Quarterly, 80(3): 528-547.

Marschall, S. (1998). Netzöffentlichkeit - eine demokratische Alternative? In Gellner, W. \& von Korff, F. (Hrsg.), Demokratie im Internet (S. 43-54). Baden-Baden: Nomos.

Mayer-Uellner, R. (2003). Das Schweigen der Lurker. München: Reinhard Fischer.

Moorstedt, T. (2008). Jeffersons Erben. Wie die digitalen Medien die Politik verändern. Frankfurt am Main: Suhrkamp.

Muhlberger, P. (2003). Political Values, Political Attitudes, and Attitude Polarization in Internet Political Discussion: Political Transformation or Politics as Usual? Communications, 28: 107-133. 
Neidhardt, F. (1994). Öffentlichkeit, Öffentliche Meinung, Soziale Bewegungen. In Neidhardt, F. (Hrsg.), Öffentlichkeit, Öffentliche Meinung, Soziale Bewegungen (S. 7-41). Opladen: Kölner Zeitschrift für Soziologie und Sozialpsychologie. Sonderheft 34.

Nie, N. H. \& Erbring, L. (2002). Internet and Society. A Preliminary Report. IT\& Society, 1(1): 275-283.

Nisbet, M. C. \& Scheufele, D. A. (2004). Political Talk as a Catalyst for Online Citizenship. Journalism \& Mass Communication Quarterly, 81(4): 877-896.

Noam, E. M. (1999). Digitaler Schwindel - Ein Hilferuf aus den USA. Online:http://www.politik-digital.de/archiv/forschung/digitalerschwindel.shtml (18.03.2010).

Norris, P. (2000). A Virtuous Circle: Political Communications in Postindustrial Societies. Cambridge, New York, Oakleigh u.a.: Cambridge University Press.

Pearson, E. (2005). Trust. Is Social Capital a Precondition for Democratic Activity Online? In Consalvo, M. \& Allen, M. (Hrsg.), Internet Research Annual: Selected Papers from the Association of Internet Researchers Conference 2003, Volume 2 (S. 85-93). New York: Peter Lang.

Peters, B. (2002). Die Leistungsfähigkeit heutiger Öffentlichkeiten - einige theoretische Kontroversen. In Imhof, K., Jarren, O. \& Blum, R. (Hrsg.), Integration und Medien (S. 23-35). Wiesbaden: Westdeutscher Verlag.

Polat, R. K. (2005). The Internet and Political Participation. Exploring the Explanatory Links. European Journal of Communication, 20(4): 435-459.

Putnam, R. D. (2000). Bowling Alone: The Collapse and Revival of American Community. New York: Simon \& Schuster.

Quandt, T. (2008). Neues Medium, alter Journalismus? Eine vergleichende Inhaltsanalyse tagesaktueller Print- und Online-Nachrichtenangebote. In Quandt, T. \& Schweiger, W. (Hrsg.), Journalismus online: Partizipation oder Profession? (S. 131-155). Wiesbaden: VS.

Rice, R. E. \& Katz, J. E. (2004). The Internet and Political Involvement in 1996 and 2000. In Howard, P. N. \& Jones, S. (Hrsg.), Society Online. The Internet in Context (S. 103-120). Thousand Oaks: Sage Publications.

Roessing, T. (2008). Opinion Formation in Wikipedia: Theory, Measurement and Findings. Masaryk University Journal of Law and Technology 2: 163-176.

Rucht, D., Yang, M. \& Zimmermann, A. (2008). Politische Diskurse im Internet und in Zeitungen. Das Beispiel Genfood. Wiesbaden: VS.

Rußmann, U. (2007). Agenda Setting und Internet. Themensetzung im Spannungsfeld von Onlinemedien und sozialen Netzwerken. München: Fischer.

Scammell, M. (2000). The Internet and Civic Engagement: The Age of the Citizen Consumer. Political Communication, 17(4): 351-356.

Scherer, H. (1998). Partizipation für alle? Die Veränderung des Politikprozesses durch das Internet. In Rössler, P. (Hrsg.), Online-Kommunikation. Beiträge zu Nutzung und Wirkung (S. 171-188). Opladen, Wiesbaden: Westdeutscher Verlag.

Schmidt, J., Paus-Hasebrink, I. \& Hasebrink, U. (2009). Heranwachsen mit dem Social Web. Zur Rolle von Web 2.0-Angeboten im Alltag von Jugendlichen und jungen Erwachsenen. Hamburg, Salzburg: Hans-Bredow-Institut für Medienforschung/Universität Salzburg.

Schönbach, K., de Waal, E. \& Lauf, E. (2005). Online and Print Newspapers: Their Impact on the Extent of the Perceived Public Agenda. European Journal of Communication, 20(2): 245-258.

Schweiger, W. \& Weihermüller, M. (2008). Öffentliche Meinung als Online-Diskurs - ein neuer empirischer Zugang. Publizistik, 53(4): 535-559. 
Shah, D. V., Schmierbach, M., Hawkins, J., Espino, R. \& Donavan, J. (2002). Nonrecursive Models of Internet Use and Community Engagement Questioning Whether Time Spent Online Erodes Social Capital. Journalism \& Mass Communication Quarterly, 79(4): 964987.

Smith, A. (2008). Post-Election Voter Engagement. Pew Internet \& American Life Project. Online: http://www.pewinternet.org/ /media//Files/Reports/2008/PIP_Voter_Engagement 2008.pdf.pdf (18.03.2010).

Smith, A. \& Rainie, L. (2008). The Internet and the 2008 Election. Pew Internet \& American Life Project. Online: http://www.pewinternet.org/ / media/Files/Reports/ 2008/PIP_Voter_ Engagement_2008.pdf(18.03.2010).

Sunstein, C. (2001). Republic.com. Princeton, NJ: Princeton University Press.

Tewksbury, D. (2006). Exposure to the News Media in a Presidential Primary Campaign. Political Communication, 23(3): 313-332.

Tewksbury, D. \& Althaus, S. L. (2000). Agenda Setting and the „New“ News. Patterns of Issue Importance Among Readers of the Paper and Online Versions of the New York Times. Communication Research, 29: 180-207.

Tsaliki, L. (2002). Online Forums and the Enlargement of Public Space: Research Findings from a European Project. Javnost - The Public, 9(2): 95-112.

Turkle, S. (1999). Looking Toward Cyberspace: Beyond Grounded Sociologoy. Contemporary Sociology, 28(6): 643-648.

Uslaner, E. M. (2004). Trust, Civic Engagement, and the Internet. Political Communication, 21(2): 223-242.

Vowe, G., Emmer, M. \& Seifert, M. (2007). Abkehr oder Mobilisierung? Zum Einfluss des Internets auf die individuelle politische Kommunikation. Empirische Befunde zu alten Fragen im Kontext neuer Medien. In Krause, B., Fretwurst, B. \& Vogelgesang, J. (Hrsg.), Fortschritte der politischen Kommunikationsforschung (S. 109-130). Wiesbaden: VS.

Wang, S.-I. (2007). Political Use of the Internet, Political Attitudes and Political Participation. Asian Journal of Communication, 17(4): 381-395.

Warnick, B. (2007). Rhetoric Online. Persuasion and Politics on the World Wide Web. New York, NY [u.a.]: Lang.

Waymer, D. (2007). Minority Opinions Go Public. Implications for Online Issues Management and the Spiral of Silence. In Duhé, S. C. (Hrsg.), New Media and Public Relations (S. 7787). New York, Frankfurt am Main u.a.: Peter Lang.

Weber, L. M., Loumakis, A. \& Bergman, J. (2003). Who Participates and Why? An Analysis of Citizens on the Internet and the Mass Public. Social Science Computer Review, 21(1): 26-42.

Winsvold, M. (2007). Municipal Websites in the Local Public Debate: Supplying Facts or Setting Agenda? NORDICOM Review, 28(2): 7-23.

Wolling, J. (2009). Onlinenutzung und Einstellungen zur Politik. Ergebnisse einer repräsentativen Panelstudie In Marcinkowski, F. \& Pfetsch, B. (Hrsg.), Politik in der Mediendemokratie: Politische Vierteljahresschrift Sonderheft 42(S. 447-467). Wiesbaden: VS.

Woodly, D. (2008). New Competencies in Democratic Communication? Blogs, Agenda Setting and Political Participation. Public Choice, 134: 109-123. 